- Francisco, California, that conducts research on emerging diseases for the US government.

In May 2011, for example, a 12-year-old girl in Uganda was admitted to hospital with a fever and bleeding from nearly every orifice. She died within hours, and doctors suspected she had been infected with a virus such as Ebola or Marburg. In the past, a conclusive diagnosis would have meant chartering a plane to airlift samples to high-containment labs across Africa or in the United States, which can take weeks. Instead, a blood sample from the girl took just three days to arrive at the Entebbe lab, which confirmed Ebola. Health-care workers reached the girl's village a day later and helped to nip a wouldbe outbreak in the bud by tracking anyone who might have been exposed (T. Shoemaker et al. Emerg. Infect. Dis. http://doi.org/h53; 2012).

Without firm diagnoses, workers tracking outbreaks have to keep tabs on thousands of suspected or potential cases. But having the
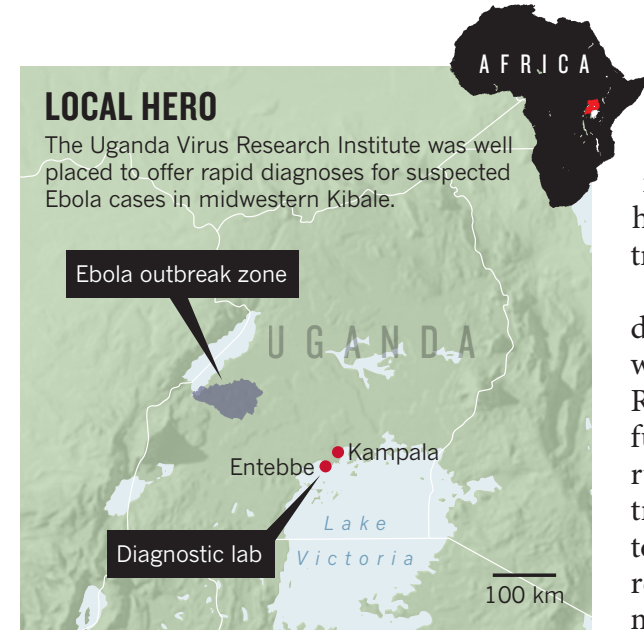

CDC lab so close to the outbreak means that "we can go back and forth to the site of the epidemic every day”, says Pierre Rollin, a virologist at the CDC's Special Pathogens Branch in
Atlanta, who is currently in Uganda. "Any samples collected this morning in Kibale will arrive at the lab and be tested tomorrow." In the latest outbreak, rapid diagnosis has meant that health-care workers had to track mere dozens of suspected cases.

"Other countries are asking us if we can develop the same kind of lab for them, but we don't have the resources to do it," says Rollin. Instead, the CDC and other Western funders support programmes to build and run laboratories in the developing world and train their staff, so that countries can monitor and respond to outbreaks with their own resources. Gabon, for example, operates a maximum biosafety-level-4 laboratory.

"You want to build capacity in the country," says Stephen Morse, an epidemiologist at Columbia University in New York, "and not wait until they ask for help to send people parachuting in."

CHEMISTRY

\title{
Amino acid provides shortcut to drugs
}

\section{Organocatalyst halves synthesis of prostaglandin family.}

\section{BY KATHARINE SANDERSON}

A $\mathrm{n}$ iconic but convoluted pharmaceutical synthesis received a major overhaul this week. The approach, described online in Nature, gives researchers easier access to a family of molecules that includes a blockbuster drug.

The efficient new route to prostaglandins, a class of hormone-like chemicals that influence processes including blood circulation and inflammation, offers a way to churn out many different versions of the molecules. It also marks a victory for one of the hottest fields in organic chemistry: organocatalysis, which speeds up chemical reactions with the help of carbonbased molecules, potentially avoiding some of the drawbacks of conventional metal catalysts.

Prostaglandin-like drugs are tempting targets for medicinal chemists; one example, Pfizer's glaucoma drug latanoprost, achieved annual sales of around US $\$ 1.75$ billion before its patent expired in 2011. But it takes an arduous 20 chemical steps to make latanoprost using the original strategy developed in 1969 by Elias Corey at Harvard University in Cambridge, Massachusetts. The synthesis helped Corey to win the Nobel Prize in Chemistry in 1990.

Synthetic chemist Varinder Aggarwal at the University of Bristol, UK, realized that he could radically simplify the process by building the heart of a prostaglandin molecule in a different way. He applied a workhorse of organic chemistry, the aldol reaction, to build key carbon-carbon bonds in a prostaglandin's fiveatom ring, but used the amino acid (S)-proline as an organocatalyst to make sure that the end product had precisely the right shape (G. Coulthard et al. Nature http://dx.doi.org/10.1038/ nature $11411 ; 2012)$. Corey's approach takes nine steps to make a different intermediate with the same crucial ring.

The molecule produced in Aggarwal's reaction serves as a foundation stone for the whole

\section{ON TARGET \\ Prostaglandin $\mathrm{PGF}_{2 \alpha}$ (below) can now be made in a seven-step synthesis that uses a simple catalyst to orient key bonds after just two steps, producing an intermediate that could generate many other prostaglandins.}

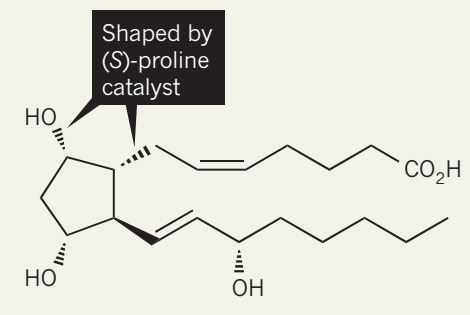

family of prostaglandins, and his method can produce grams of this building block at a time - a viable scale for drug-development work. Aggarwal and his colleagues used it to make the most complicated prostaglandin, $\mathrm{PGF}_{2 \alpha}$, which is very similar to latanoprost, in just seven steps (see 'On target'). It's "by far the single best approach to the prostaglandins", says Ben List, an expert in organocatalysis at the Max Planck Institute for Coal Research in Mülheim an der Ruhr, Germany. As well as being shorter than Corey's version, it is very specific at producing only left-handed or right-handed versions of the molecules - a crucial factor in making an effective drug.

Whether the synthesis helps to deliver new drugs remains to be seen, says biomedical researcher Colin Funk at Queen's University in Kingston, Ontario, Canada. "Prostaglandinlike drugs are still relevant, but the big thing has been to try and limit their side effects," he says. That demands a better biological understanding, rather than a new synthetic route, he notes.

The method might prove useful in full-scale drug production, not only because of its simplicity but because it avoids expensive and toxic metal catalysts. "From an industrial perspective Aggarwal's process is promising, and a remarkable lead," says process chemist Rob Singer, who works at Pfizer Worldwide Research and Development in Groton, Connecticut. The synthesis would still need further development before it could be used at a production scale, he adds.

List says that most pharmaceutical companies are studying organocatalysts, but so far have tended to use them on only a small scale. As the chemical structures of drugs get more complicated, he hopes that organocatalysts will gain more prominence, and Aggarwal's work should help to speed the change, he says. "It reflects the way that organocatalysis fundamentally changes the way we do catalysis," he says. 